\title{
Angiotensin II protects cortical neurons against oxygen-glucose deprivation-induced injury in vitro
}

\author{
MINGTAN TANG* , LI ZHAO ${ }^{*}$, YANQING CHEN, LIXIANG WANG and XIUMEI ZHANG \\ Department of Pharmacology, Shandong University School of Medicine, Jinan, Shandong 250012, P.R. China
}

Received September 27, 2013; Accepted October 02, 2013

DOI: $10.3892 /$ br.2013.182

\begin{abstract}
Ischemic cerebrovascular disease is a common type of cerebrovascular disease and the leading cause of disability and mortality worldwide. Therefore, it is crucial to elucidate its pathogenesis and develop novel therapeutic strategies. This study was performed to investigate whether angiotensin (Ang) II exerts a protective effect against cerebral ischemia/reperfusion (I/R) injury in vitro. The primary cultured neurons were prepared and an I/R model was established by incubation of cortical neurons with $\mathrm{Na}_{2} \mathrm{~S}_{2} \mathrm{O}_{4}$, followed by culture in fresh medium. The protective effect of Ang II and its underlying mechanisms were investigated by morphology observation, MTT assay, flow cytometry analysis and reverse transcription-polymerase chain reaction (RT-PCR). The data demonstrated that Ang II significantly ameliorated the neuronal injury caused by oxygen-glucose deprivation. Furthermore, Ang II increased cell viability through inhibiting cell apoptosis. The RT-PCR results revealed that Ang II was able to reverse the increased bax mRNA and the decreased $b c l 2$ mRNA expression. Of note, the protective activity of Ang II may be attenuated by co-treatment with Ang II type 2 (AT2) receptor blockade (PD123319), but not Ang II type 1 (AT1) receptor blockade (valsartan). These findings suggested that Ang II exerted a protective effect against neuronal injury induced by oxygen-glucose deprivation through decreasing cell apoptosis. Therefore, Ang II may be used as a potential therapeutic target in the future.
\end{abstract}

\section{Introduction}

Ischemic cerebrovascular disease is currently one of the leading causes of mortality and long-term disability worldwide, often resulting in irreversible brain damage and subsequent loss of neuronal function $(1,2)$. The pathophysiological mechanisms underlying initial and secondary injury following ischemia

Correspondence to: Dr Xiumei Zhang, Department of Pharmacology, Shandong University School of Medicine, 44 Wenhua Xi Road, Jinan, Shandong 250012, P.R. China

E-mail: zhangxm@sdu.edu.cn

*Contributed equally

Key words: angiotensin II, ischemia/reperfusion injury, apoptosis have not been fully elucidated. Therefore, it is crucial to investigate the pathogenesis of cerebral ischemia and develop novel, effective methods to prevent and/or treat this disease.

It is well known that cell apoptosis is crucial in neuronal death (3). Currently available evidence demonstrates that apoptosis is involved in the injury associated with ischemic cerebral diseases, particularly cerebral ischemia/reperfusion (I/R) injury $(4,5)$. Due to its complexity, the precise mechanism of apoptosis induced by cerebral ischemia remains unclear, although bcl2 and bax were shown to play important roles (5). $\mathrm{Bcl} 2$ and bax belong to bcl2-related protein subfamilies, which are encoded by several genes affecting cell apoptosis, among which the $b c l 2$ gene was shown to act as an anti-apoptotic factor and the bax gene as a promoter of apoptosis $(6,7)$. Novel drugs, which may decrease the bax and/or increase the $b c l 2$ gene expression, may exert a protective effect against neuronal apoptosis induced by I/R injury. Indeed, estradiol, erythropoietin and SP600125 were reported to inhibit ischemia-induced neuronal apoptosis through the regulation of $b c l 2$ and/or bax gene expression (8-10).

Angiotensin (Ang) II is produced from Ang I through removal of two C-terminal residues by the angiotensin-converting enzyme. Ang II is best known for its role in the regulation of blood pressure, fluid balance and neuroendocrine function (11-13). It was recently demonstrated that Ang II primarily activated circumventricular neurons, leading to the activation of neurons in other forebrain regions (14), indicating that Ang II may exert multiple effects on neuronal activity. It was also recently suggested that Ang II exerts a protective effect against cortical neuron injury induced by hypoxia (15). In this study, an in vitro ischemia-induced cell model was established to investigate whether Ang II exerts a protective effect against neuronal injury and its mechanism of action.

\section{Materials and methods}

Materials. Ang II, PD123319 and valsartan were purchased from Sigma (St. Louis, MO, USA). Cytarabine hydrochloride was purchased from Changzhou Lab market Co. (Changzhou, China). $\mathrm{Na}_{2} \mathrm{~S}_{2} \mathrm{O}_{4}$ was purchased from Tianjin Chemical Reagents Co., Inc. (Xiqing, Tianjin, China). TRIzol was purchased from the Shanghai Bioengineering Institute (Shanghai, China). The Annexin V-propidium iodide (PI) apoptosis kit was purchased from Shenzhen Jingmei Biotechnology Co., Ltd. (Shenzhen, Guangdong, China). 
A

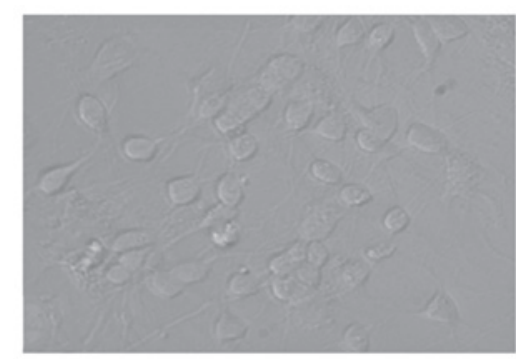

C

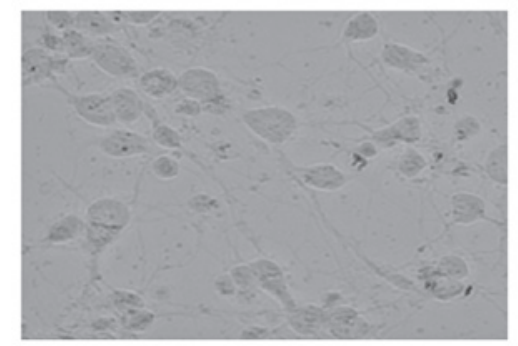

B

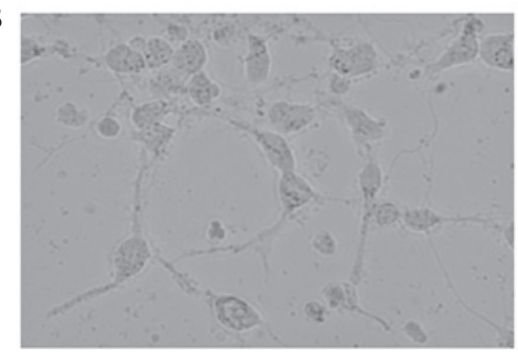

D

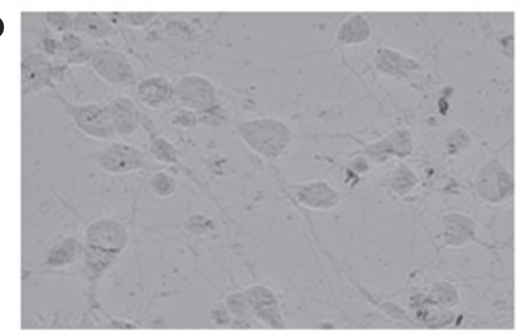

$\mathbf{E}$

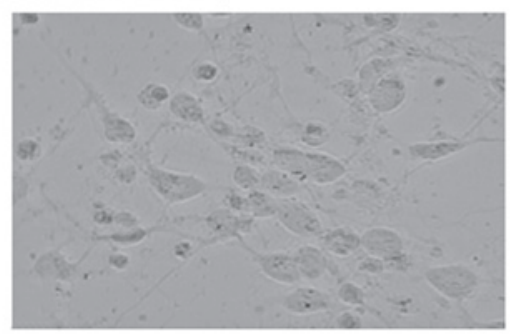

Figure 1. Effect of angiotensin II (Ang II) on neuronal damage induced by ischemia/reperfusion (I/R) injury. The primary cortical neurons were prepared and an in vitro $\mathrm{I} / \mathrm{R}$ injury model was established. Following treatment with the selected substances for $12 \mathrm{~h}$, cell morphology was observed and representative pictures are presented (magnification, $\mathrm{x} 400)$. A, normal group; B, model group; $\mathrm{C}$, Ang II (1 $\mu \mathrm{M})$ group; D, Ang II (1 $\mu \mathrm{M})$ plus PD123319 (10 $\mu \mathrm{M})$ group; and E, Ang II $(1 \mu \mathrm{M})$ plus valsartan $(10 \mu \mathrm{M})$ group.

Animals. Male and female Wistar rats (weighing 220-250 g) were obtained from the Laboratory Animal Center of Shandong University. The animals were housed in a light- and temperature-controlled room $\left(21-22^{\circ} \mathrm{C}\right.$, humidity $\left.50-65 \%\right)$ and maintained on a standard diet and water. All the experiments were performed in accordance with the Shandong University Guide for the Care and Use of Laboratory Animals.

Primary neuronal cell culture. The primary neuronal cell cultures were performed as previously described, with minor modifications (16). Briefly, the neonatal brains were removed and dissected in D-Hank's solution. After stripping the meninges and blood vessels, the brain tissue was minced into $1-\mathrm{mm}^{3}$ pieces and trypsinized at $37^{\circ} \mathrm{C}$ for $25 \mathrm{~min}$. The cells were seeded into 96 - or 12 -well plates pre-coated with poly-D-lysine and maintained at $37^{\circ} \mathrm{C}$ in a humidified atmosphere containing $5 \% \mathrm{CO}_{2}$. Cystine arabinofuranoside was added to the medium to inhibit the growth of cells other than neurons.

In vitro ischemia/reperfusion $(I / R)$ injury model and morphology observation. The experiments were conducted on 7-day-old primary cultured cortical neurons. Briefly, the primary cultured neurons were first incubated with $\mathrm{Na}_{2} \mathrm{~S}_{2} \mathrm{O}_{4}$ at a concentration of $1 \mathrm{mM}$ in sugar-free Earle's solution for $1 \mathrm{~h}$. The cell solution was then replaced by fresh medium, in which the in vitro I/R model was established. Five groups were then designed as follows: the control group, in which cortical neurons were incubated with normal medium; the model group, in which the solution was replaced with normal cell culture medium; the Ang II group, in which the medium contained Ang II at a concentration of $1 \mu \mathrm{M}$; the Ang II plus PD123319 group, in which the medium contained Ang II at a concentration of $1 \mu \mathrm{M}$ and PD123319 at a concentration of $10 \mu \mathrm{M}$; and the Ang II plus valsartan group, in which the medium contained Ang II at a concentration of $1 \mu \mathrm{M}$ and valsartan at a concentration of $10 \mu \mathrm{M}$. Following incubation for $12 \mathrm{~h}$, the cell morphology was observed under an inverted phase contrast microscope and representative images were selected.

Cell viability. The cell viability was measured with the MTT assay, as previously described (17). Briefly, the cortical neurons were incubated as dictated by the experiment design for $12 \mathrm{~h}$ and the medium was then replaced by MTT solution ( $200 \mu \mathrm{g} / \mathrm{ml}$ in normal medium) for another $2 \mathrm{~h}$. The medium was aspirated and the formazan dye crystals were dissolved in $200 \mu \mathrm{l}$ dimethyl sulfoxide. The cell viability was determined by optical density values at $570 \mathrm{~nm}$ measured by an automatic plate reader.

Annexin $V$ staining. The Annexin V staining assay was performed according to the manufacturer's protocol. Briefly, the cells were washed twice with D-Hank's solution after treatment and digested with trypsin. The cells were collected by centrifugation at $300 \mathrm{x}$ g for $5 \mathrm{~min}$ and suspended and the density was adjusted to $5 \times 10^{5}$ cells $/ \mathrm{ml}$, in which $195 \mu \mathrm{l}$ cell suspension and $5 \mu \mathrm{l}$ Annexin V-fluorescein isothiocyanate 
Table I. Primer sequences for $b c l 2$, bax and $\beta$-actin.

\begin{tabular}{lll}
\hline Gene & \multicolumn{1}{c}{ Sequences } & $\begin{array}{c}\text { Product } \\
\text { length }\end{array}$ \\
\hline Bcl2 & F: 5'-CTGGTGGACAACATCGCTCTG-3' & 227 bp \\
& R: 5'-GGTCTGCTGACCTCACTTGTG-3' & \\
Bax & F: 5'-GCAGAGGATGATTGCTGATG-3' & 354 bp \\
& R: 5'-CTCAGCCCATCTTCTTCCAG-3' & \\
$\beta$-actin & F: 5'-TTGGCACCACACTTTCTACA-3' & $380 \mathrm{bp}$ \\
& R: 5'-TCACGCACGATTTCCCTCTCAG-3' & \\
\hline
\end{tabular}

F, forward; R, reverse.

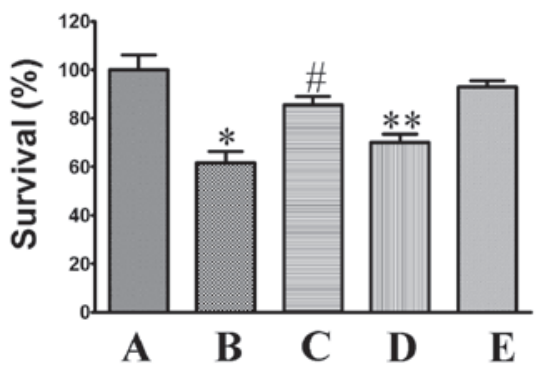

Figure 2. Effect of angiotensin II (Ang II) on neuronal viability following ischemia/reperfusion (I/R) injury. The primary cortical neurons were prepared and an in vitro I/R injury model was established. Following treatment with the selected substances for $12 \mathrm{~h}$, the cell viability was measured with the MTT assay and the relative survival was calculated. A, normal group; B, model group; C, Ang II $(1 \mu \mathrm{M})$ group; D, Ang II $(1 \mu \mathrm{M})$ plus PD123319 $(10 \mu \mathrm{M})$ group; and $\mathrm{E}$, Ang II $(1 \mu \mathrm{M})$ plus valsartan $(10 \mu \mathrm{M})$ group. " $\mathrm{P}<0.05$, compared to the control group; ${ }^{\text {}} \mathrm{P}<0.05$, compared to the model group; ${ }^{* *} \mathrm{P}<0.05$, compared to the Ang II group.

were mixed and incubated for $10 \mathrm{~min}$ at room temperature. After washing, the cells were resuspended in $190 \mu \mathrm{l}$ pre-diluted binding buffer and stained with PI $(1 \mu \mathrm{g} / \mathrm{ml})$. The cells were finally analysed by flow cytometry (FCM) and the apoptosis ratios were calculated.

Reverse transcription-polymerase chain reaction (RT-PCR) analysis. Total mRNA was prepared and RT-PCR was performed as previously described (18). Briefly, the cells were washed twice with D-Hank's solution after treatment and total RNA was extracted from the cultured cortical neurons using a TRIzol extraction kit. After RT, the PCR assay was performed to amplify the $b c l 2$, bax and $\beta$-actin. The PCR products were then subjected to $2 \%$ agarose gel electrophoresis and visualized by staining with ethidium bromide. The optical density of each band was measured using ImageJ software and the semi-quantitative measure was expressed as a ratio compared to that of $\beta$-actin. The primer sequences for $b c l 2$, bax and $\beta$-actin are presented in Table I.

Statistical analysis. Data are expressed as values \pm SD and analyzed by one-way ANOVA. The difference between two groups was assessed by the Student's t-test. $\mathrm{P}<0.05$ was considered to indicate a statistically significant difference between any two groups.
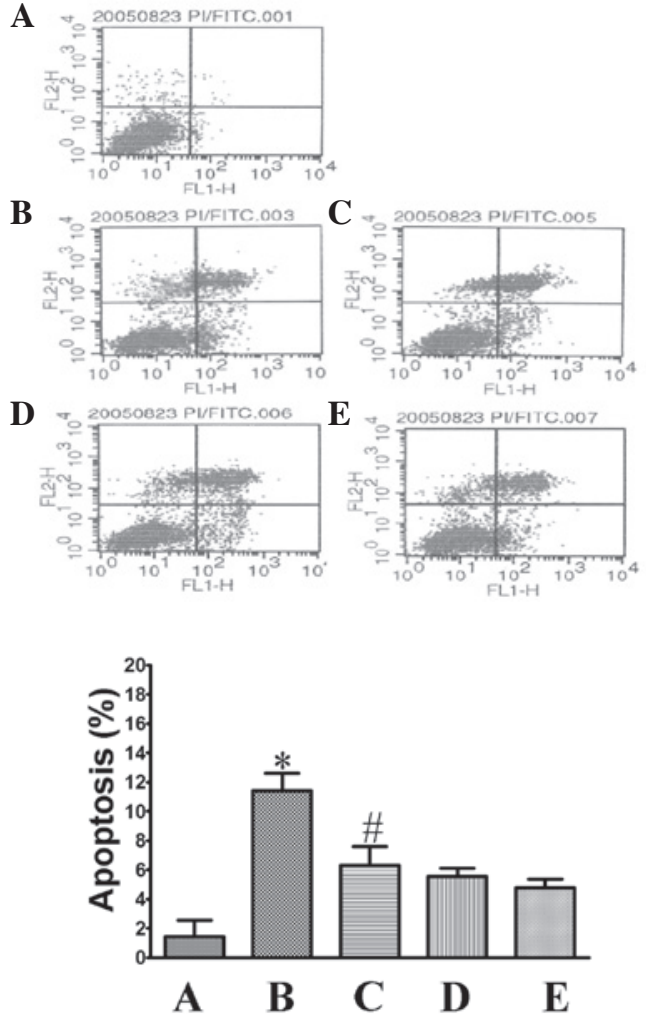

Figure 3. Effect of angiotensin II (Ang II) on cell apoptosis following ischemia/reperfusion (I/R) injury. The primary cortical neurons were prepared and an in vitro I/R injury model was established. Following treatment with the selected substances for $12 \mathrm{~h}$, the cells were assessed with Annexin V staining assay and analysed by flow cytometry and the apoptosis ratio was calculated. A, normal group; B, model group; C, Ang II (1 $\mu \mathrm{M})$ group; D, Ang II $(1 \mu \mathrm{M})$ plus PD123319 $(10 \mu \mathrm{M})$ group; and E, Ang II $(1 \mu \mathrm{M})$ plus valsartan $(10 \mu \mathrm{M})$ group. ${ }^{*} \mathrm{P}<0.05$, compared to the control group; ${ }^{\sharp} \mathrm{P}<0.05$, compared to the model group.

\section{Results}

Ang II attenuates the I/R-induced neuronal damage. Following observation under an inverted phase contrast microscope, the primary cultured neurons were characterized morphologically and biochemically (Fig. 1). The cells in the model group appeared swollen, with the neurites being contracted or even severed. However, the cells in the Ang II group appeared to to exhibit a reconstituted morphology. Similar morphological characteristics were observed in the presence of Ang II plus valsartan or PD123319, with Ang II plus valsartan exerting a more potent protective effect.

Ang II increases the viability of oxygen-glucose deprivation-treated neurons. MTT was commonly used to assess cell viability. As shown in Fig. 2, I/R injury significantly reduced cell viability to $61.65 \pm 4.67 \%(\mathrm{P}<0.05$, compared to that in the control group). However, incubation with Ang II significantly attenuated cell death to $85.55 \pm 3.54 \%$ ( $\mathrm{P}<0.05$, compared to that in the model group). The protective activity of Ang II was significantly attenuated by co-incubation with PD123319, with the relative survival being reduced to $70.10 \pm 3.38 \%(\mathrm{P}<0.05$, compared to that in the Ang II group). Co-treatment with valsartan was shown to further increase the protective activity of Ang II to $92.95 \pm 2.26 \%$. 

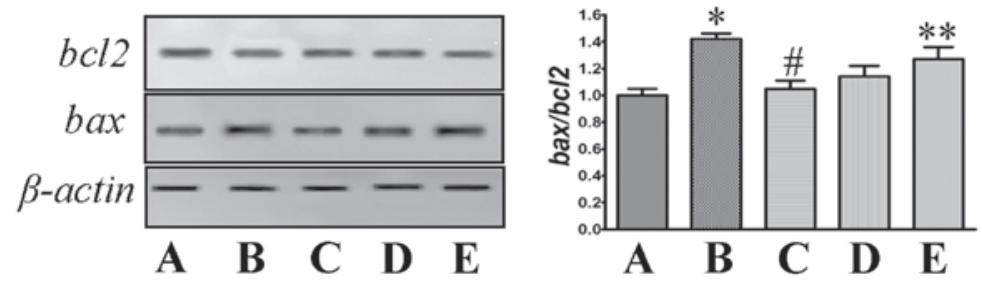

Figure 4. Effect of angiotensin II (Ang II) on $b c l 2$ and bax mRNA expression in neurons following ischemia/reperfusion (I/R) injury. The primary cortical neurons were prepared and an in vitro I/R injury model was established. Following treatment with the selected substances for $12 \mathrm{~h}$, total mRNA was prepared and polymerase chain reaction (PCR) was performed after reverse transcription. The PCR products were subjected to gel electrophoresis and the optical density of each band was measured by ImageJ software. A, normal group; B, model group; C, Ang II ( $1 \mu \mathrm{M})$ group; D, Ang II (1 $\mu \mathrm{M})$ plus valsartan (10 $\mu \mathrm{M})$ group; and E, ang II $(1 \mu \mathrm{M})$ plus PD123319 $(10 \mu \mathrm{M})$ group. ${ }^{*} \mathrm{P}<0.05$, compared to the control group; ${ }^{~} \mathrm{P}<0.05$, compared to the model group; ${ }^{* *} \mathrm{P}<0.05$, compared to the Ang II group.

Ang II attenuates cell apoptosis induced by oxygen-glucose deprivation and reperfusion. The Annexin V staining assay was used to detect cell apoptosis by FCM. As shown in Fig. 3, the apoptosis ratio in the control group was $1.44 \pm 1.11 \%$. After the cells were exposed to oxygen-glucose deprivation and reperfusion, that ratio increased to $11.4 \pm 1.20 \%(\mathrm{P}<0.05$, compared to that in the control group), which indicated that the cells were undergoing apoptosis. Treatment of the cells with Ang II significantly attenuated cell apoptosis, with an apoptosis ratio of $5.27 \pm 0.55 \%(\mathrm{P}<0.05$, compared to that in the model group). Co-treatment with PD123319 or valsartan was shown to inhibit cell apoptosis with a similar tendency (apoptosis ratio, $5.55 \pm 0.58$ and $4.77 \pm 0.62 \%$, respectively).

Ang II increases the ratio of bcl2/bax in mRNA expression. Total mRNA was prepared and RT-PCR was performed. As shown in Fig. 4, the model group exhibited increased mRNA expression of bax and decreased mRNA expression of $b c l 2$, with a significant increase of the bcl2/bax ratio $(\mathrm{P}<0.05$, compared to that in the control group). Co-incubation with Ang II attenuated the increased bax and decreased $b c l 2$, which reduced the changes in the $b c l 2 / b a x$ ratio $(\mathrm{P}<0.05$, compared to that in the model group). Co-treatment with PD123319 or valsartan exhibited a similar tendency.

\section{Discussion}

The cerebral vascular event (stroke) caused by ischemia or hemorrhage is the most common type of cerebrovascular disease and the main cause of disability and mortality worldwide (1). Since the pathophysiological mechanism of initial and secondary injury after ischemia has not been fully elucidated, it is crucial to investigate its pathogenesis and develop novel treatment methods.

The central nervous system is vulnerable to the effects of disease and toxic or metabolic insults, such as oxygen and glucose deprivation, which often occurs during stroke and cardiac arrest $(19,20)$. Although thrombolysis has been approved as an effective therapy for acute ischemic stroke with specific time windows, early reperfusion may aggravate edema formation (21). In this study, $\mathrm{Na}_{2} \mathrm{SO}_{4}$ was used to establish the hypoxic environment through clearing oxygen from the culture medium, followed by medium re-incubation, which mimics the process of cerebral I/R injury. Our data clearly demonstrated that the I/R injured the primary cultured neurons, which were observed to be swollen, with the neurites being contracted, or even severed. Incubation with Ang II was able to reconstitute the morphology of the injured cells, which indicated that Ang II exerted a protective effect against I/R-induced injury in neurons. Consistently, I/R injury significantly decreased cell survival, as was detected by the MTT assay. Treatment with Ang II was able to attenuate cell death induced by I/R injury, which also indicated that Ang II may be used against ischemic cerebral vascular disease.

It is well known that neuronal death is associated with apoptosis and currently available evidence demonstrates that apoptosis is involved in the injury associated with ischemic cerebral diseases, particularly cerebral ischemia/reperfusion (I/R) injury $(3,4)$. Apoptosis may be the response to a cellular 'insult', such as chemical or physical damage. The insult initiates a cascade of events leading to DNA breaks and the destruction of the cell, which are easily detected and analyzed by several methods, such as the TUNEL staining assay, electron microscope scanning and Annexin V staining $(22,23)$. In this study, we performed the Annexin V staining assay and analyzed the apoptosis ratio in neurons with flow cytometry. Our data demonstrated that I/R injury induced neuronal apoptosis, as indicated by the ratio of PI staining-positive cells. Treatment with Ang II attenuated cell apoptosis induced by the ischemic stress, which indicated that the protective effect of Ang II may be partially mediated though preventing the neurons from undergoing apoptosis.

The mechanism of apoptosis induced by ischemic injury in neurons has not been fully elucidated, although the bcl2 family was shown to play an important role (6). Based on structural and functional characteristics, the protein families were divided into anti-apoptotic members, such as bcl2, and pro-apoptotic members, such as bax (7). After the neurons were exposed to I/R injury, the mRNA levels of bax were observed to increase and the mRNA levels of $b c l 2$ were decreased. The disruption of balance between anti- and pro-apoptotic effects induced cell apoptosis. However, treatment with Ang II was shown to increase the reduced $b c l 2$ expression and decrease the elevated bax expression, which in turn attenuated the neuronal apoptosis induced by I/R injury.

Ang II is best known for its role in the regulation of cardiovascular behavior and fluid homeostasis, through binding with its receptors, of which there were two major isoforms, Ang II type 1 (AT1) receptor and AT2 receptor $(24,25)$. These isoforms are considered to account for the diverse actions of Ang II in peripheral organs, as well as in the nervous system. Based on our results, the protective effect of Ang II against neuronal 
death induced by I/R injury may be inhibited by co-incubation with PD123319, but not with valsartan, indicating that this protective effect of Ang II was exerted in an AT2-dependent manner. Of note, co-treatment with valsartan did not decrease, but rather increased cell survival. This finding was consistent with previous findings stating that chronic blockade of AT1 receptors with losartan prevented bax upregulation and normalized apoptosis in these cells, independently of its hemodynamic effect (26). The exact molecular mechanism through which Ang II displays its protective activity and its effects in vivo remains unclear and requires further investigation.

In summary, to the best of our knowledge, this was the first study to demonstrate that Ang II exerts a protective effect against ischemia-induced neuronal injury in an AT2-dependent manner, which is associated with the inhibition of neuronal apoptosis via the regulation of the ratio of bax/bcl2 mRNA expression by Ang II. Therefore, Ang II may be used as a therapeutic target in the future.

\section{References}

1. Read SJ, Hirano T, Davis SM and Donnan GA: Limiting neurological damage after stroke: a review of pharmacological treatment options. Drugs Aging 14: 11-39, 1999.

2. Baron JC: Perfusion thresholds in human cerebral ischemia: historical perspective and therapeutic implications. Cerebrovasc Dis 11 (Suppl 1): 2-8, 2001.

3. Okouchi M, Ekshyyan O, Maracine M and Aw TY: Neuronal apoptosis in neurodegeneration. Antioxid Redox Signal 9: 1059-1096, 2007.

4. Broughton BR, Reutens DC and Sobey CG: Apoptotic mechanisms after cerebral ischemia. Stroke 40: e331-e339, 2009.

5. Niizuma K, Yoshioka H, Chen $\mathrm{H}$, et al: Mitochondrial and apoptotic neuronal death signaling pathways in cerebral ischemia. Biochim Biophys Acta 1802: 92-99, 2010.

6. Shamas-Din A, Kale J, Leber B and Andrews DW: Mechanisms of action of Bcl-2 family proteins. Cold Spring Harb Perspect Biol 5: a008714, 2013.

7. Youle RJ and Strasser A: The BCL-2 protein family: opposing activities that mediate cell death. Nat Rev Mol Cell Biol 9: 47-59, 2008.

8. Ayaloglu-Butun F, Terzioglu-Kara E, Tokcaer-Keskin Z and Akcali KC: The effect of estrogen on bone marrow-derived rat mesenchymal stem cell maintenance: inhibiting apoptosis through the expression of Bcl-xL and Bcl-2. Stem Cell Rev 8: 393-401, 2012.

9. Warren JS, Zhao Y, Yung R and Desai A: Recombinant human erythropoietin suppresses endothelial cell apoptosis and reduces the ratio of $\mathrm{Bax}$ to $\mathrm{Bcl}-2$ proteins in the aortas of apolipoprotein E-deficient mice. J Cardiovasc Pharmacol 57: 424-433, 2011.

10. Ramin M, Azizi P, Motamedi F, Haghparast A and Khodagholi F: Inhibition of JNK phosphorylation reverses memory deficit induced by $\beta$-amyloid (1-42) associated with decrease of apoptotic factors. Behav Brain Res 217: 424-431, 2011.
11. Wysocki J, Ye M, Rodriguez E, et al: Targeting the degradation of angiotensin II with recombinant angiotensin-converting enzyme 2: prevention of angiotensin II-dependent hypertension. Hypertension 55: 90-98, 2010.

12. Reis WL, Saad WA, Camargo LA, Elias LL and Antunes-Rodrigues J: Central nitrergic system regulation of neuroendocrine secretion, fluid intake and blood pressure induced by angiotensin-II. Behav Brain Funct 6: 64, 2010.

13. Grobe JL, Grobe CL, Beltz TG, et al: The brain renin-angiotensin system controls divergent efferent mechanisms to regulate fluid and energy balance. Cell Metab 12: 431-442, 2010.

14. Cao X, Peterson JR, Wang G, et al: Angiotensin II-dependent hypertension requires cyclooxygenase 1-derived prostaglandin E2 and EP1 receptor signaling in the subfornical organ of the brain. Hypertension 59: 869-876, 2012.

15. McCarthy CA, Vinh A, Broughton BR, Sobey CG, Callaway JK and Widdop RE: Angiotensin II type 2 receptor stimulation initiated after stroke causes neuroprotection in conscious rats. Hypertension 60: 1531-1537, 2012.

16. Pont-Lezica L, Colasse S and Bessis A: Depletion of microglia from primary cellular cultures. Methods Mol Biol 1041: 55-61, 2013.

17. Wang $\mathrm{H}, \mathrm{Yu}$ P, Gou $\mathrm{H}$, et al: Cardioprotective effects of 20(S)-ginsenoside Rh2 against doxorubicin-induced cardiotoxicity in vitro and in vivo. Evid Based Complement Alternat Med 2012: 506214, 2012.

18. Wang H, Yu P, Bai J, et al: Ocotillol enhanced the antitumor activity of doxorubicin via p53-dependent apoptosis. Evid Based Complement Alternat Med 2013: 468537, 2013.

19. Xilouri M and Stefanis L: Autophagy in the central nervous system: implications for neurodegenerative disorders. CNS Neurol Disord Drug Targets 9: 701-719, 2010.

20. Zhou T, Jiang J, Zhang M, Fu Y, Yang Z and Jiang L: Protective effect of mild hypothermia on oxygen-glucose deprivation injury in rat hippocampal neurons after hypoxia. Mol Med Rep 7: 1859-1864, 2013.

21. Dominguez C, Delgado P, Vilches A, et al: Oxidative stress after thrombolysis-induced reperfusion in human stroke. Stroke 41: 653-660, 2010.

22. Zhang X, Chen Y, Jenkins LW, Kochanek PM and Clark RS: Bench-to-bedside review: Apoptosis/programmed cell death triggered by traumatic brain injury. Crit Care 9: 66-75, 2005.

23. Krysko DV, Vanden Berghe T, D'Herde K and Vandenabeele P: Apoptosis and necrosis: detection, discrimination and phagocytosis. Methods 44: 205-221, 2008.

24. Kaschina E and Unger T: Angiotensin AT1/AT2 receptors: regulation, signalling and function. Blood Press 12: 70-88, 2003.

25. Goldenberg I, Grossman E, Jacobson KA, Shneyvays V and Shainberg A: Angiotensin II-induced apoptosis in rat cardiomyocyte culture: a possible role of AT1 and AT2 receptors. J Hypertens 19: 1681-1689, 2001.

26. Fortuno MA, Ravassa S, Etayo JC and Diez J: Overexpression of Bax protein and enhanced apoptosis in the left ventricle of spontaneously hypertensive rats: effects of AT1 blockade with losartan. Hypertension 32: 280-286, 1998. 